\title{
Inhibition of Tau Aggregation by Three Aspergillus nidulans Secondary Metabolites: 2, $\omega$-Dihydroxyemodin, Asperthecin, and Asperbenzaldehyde
}

Authors

Affiliations
Smita R. Paranjape ${ }^{1}$, Yi-Ming Chiang ${ }^{2,3}$, James F. Sanchez ${ }^{2}$, Ruth Entwistle ${ }^{1}$, Clay C. C. Wang ${ }^{2,4}$, Berl R. Oakley ${ }^{1}$, T. Chris Gamblin

The affiliations are listed at the end of the article
Key words

- tau

- microtubule-associated protein

- Trichocomaceae

- Aspergillus nidulans

- Alzheimer's disease received June 28,2013

revised October 25, 2013

accepted Nov. 22, 2013

Bibliography

Dol http://dx.doi.org/

10.1055/s-0033-1360180

Planta Med 2014; 80: 77-85

(c) Georg Thieme Verlag KG

Stuttgart - New York .

ISSN 0032-0943

\section{Correspondence}

Assoc. Prof. T. Chris Gamblin

Department of Molecular

Biosciences

University of Kansas

1200 Sunnyside Ave.

Lawrence, KS 66045

United States

Phone: + 17858645065

Fax: + 17858645321

gamblin@ku.edu

\section{Abstract \\ $\nabla$}

The aggregation of the microtubule-associated protein tau is a significant event in many neurodegenerative diseases including Alzheimer's disease. The inhibition or reversal of tau aggregation is therefore a potential therapeutic strategy for these diseases. Fungal natural products have proven to be a rich source of useful compounds having wide varieties of biological activity. We have screened Aspergillus nidulans secondary metabolites containing aromatic ring structures for their ability to inhibit tau aggregation in vitro using an arachidonic acid polymerization protocol and the previously identified aggregation inhibitor emodin as a positive control. While several compounds showed some activity, 2, $\omega$-dihydroxyemodin, asperthecin, and asperbenzaldehyde were potent aggregation inhibitors as determined by both a filter trap assay and electron microscopy. In this study, these three compounds were stronger inhibitors than emodin, which has been shown in a prior study to inhibit the heparin

\section{Introduction}

\section{$\nabla$}

Alzheimer's disease and other neurodegenerative tauopathies are characterized by the abnormal accumulation of aggregated forms of the microtubule associated protein tau. Tau aggregation correlates with the type and severity of cognitive impairment in Alzheimer's disease [1]; mutations in the tau gene can lead to neurodegeneration $[2,3]$, and tau aggregation can lead to cell death and cognitive defects in cellular and animal models (reviewed in [4]). It is therefore believed that inhibiting tau aggregation is a viable therapeutic target for the treatment of Alzheimer's disease and related tauopathies $[5,6]$.

Tau aggregation is caused when natively unfolded sequences in their microtubule-binding repeats 3 induction of tau aggregation with an $\mathrm{IC}_{50}$ of 1 $5 \mu \mathrm{M}$. Additionally, 2, $\omega$-dihydroxyemodin, asperthecin, and asperbenzaldehyde reduced, but did not block, tau stabilization of microtubules. 2, $\omega$ Dihydroxyemodin and asperthecin have similar structures to previously identified tau aggregation inhibitors, while asperbenzaldehyde represents a new class of compounds with tau aggregation inhibitor activity. Asperbenzaldehyde can be readily modified into compounds with strong lipoxygenase inhibitor activity, suggesting that compounds derived from asperbenzaldehyde could have dual activity. Together, our data demonstrates the potential of 2, $\omega$-dihydroxyemodin, asperthecin, and asperbenzaldehyde as lead compounds for further development as therapeutics to inhibit tau aggregation in Alzheimer's disease and neurodegenerative tauopathies.

Supporting information available online at http://www.thieme-connect.de/ejournals/toc/ plantamedica

and 4 undergo a conformational change, potentially as a result of phosphorylation [7-9], to $\beta$ sheet structure $[10,11]$ and then interact with one another to form amyloid-type filaments. It is believed that small molecules that interact with the $\beta$-sheet structure inhibit tau aggregation by preventing the addition of tau molecules to the growing aggregate. Tau aggregation inhibitors identified to date belong to chemical classes including phenothiazines [12,13], cyanine dyes $[14,15]$, polyphenols [12], pthalocyanines [12], anthraquinones [16], N-phenylamines [17], phenylthiazolyl-hydrazides [18], rhodanines [19], quinoxalines [20], and thienopyridazines [21]. These compounds represent a wide variety of chemical structures, but they share the common feature of multiple aromatic rings. While it is not 


\section{Multicyclic Aromatics}

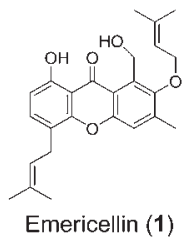

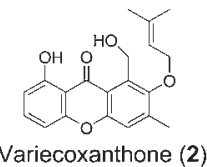<smiles>CCOc1cc(C)cc(O)c1O</smiles><smiles>O=C1c2cc(O)cc(O)c2C(=O)c2c(O)c(O)cc(O)c21</smiles>

2,(0-Dihydroxyemodin (4)<smiles>Cc1cc2c(c(O)c1C(=O)O)C(=O)c1c(O)cc(O)cc1C2=O</smiles>

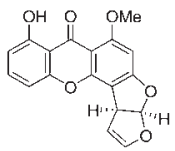

Endocrocin (5) Sterigmatocystin (6)

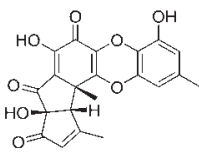

F9775 A (7)<smiles></smiles><smiles>O=C1c2cc(CO)cc(O)c2C(=O)c2cccc(O)c21</smiles>

Chrysophanol (10)

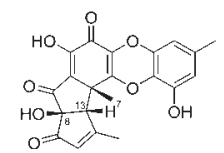

F9775 B (8)<smiles>OCc1c(O)c(O)c2c(O)c(O)cc(O)c2c1O</smiles>

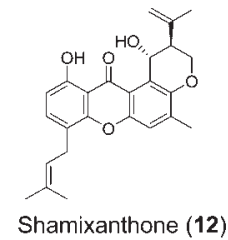

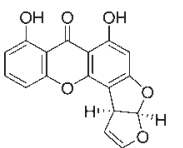

Demethylsterigmatocystin (13)<smiles>O=C1c2cc(O)cc(O)c2C(=O)c2c(O)cc(O)cc21</smiles>
(a)-Hydroxyemodin (14) Monodictyphenone (15)

Fig. 1 Compounds used in this study. The chemical structures are drawn for the 17 compounds used. The names of the compounds are included with their structure along with a compound number. Each compound was purified from a single HPLC peak. The purity of each compound was estimated from its ${ }^{1} \mathrm{H}$ NMR spectrum (see Supporting Information) and is listed in Table 1S. In F9775 B, NOESY correlations between $\mathrm{H}-13 / \mathrm{H}_{3}-7$ and $\mathrm{H}-13 /$ $\mathrm{OH}-8$ suggested that $\mathrm{H}_{3}-7, \mathrm{OH}-8$, and $\mathrm{H}-13$ are on the same face. The fact that the specific rotation of F9775 A and B is close to zero indicated that both compounds are racemic mixtures [48]. Stereocenters on the side chain of $3^{\prime}$-hydroxyversiconol have not been determined due to the free rotation of the side chain.

\section{Monocyclic Aromatic}

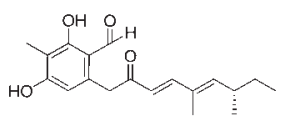

Asperbenzaldehyde (17)

known whether these inhibitors will be effective in vivo or whether they have suitable bioavailability or pharmacokinetic properties to serve this purpose, it is important to have lead compounds with the appropriate biological activity for further development.

Fungal natural products and secondary metabolites have historically been a rich source of compounds with useful biological activities such as antibiotics, antimicrobials, and antioxidants. Recent advances in genetics and genomics have greatly facilitated the study of fungal metabolic pathways along with the identification and purification of biologically interesting compounds. Using an efficient gene targeting system [22-24], we have identified several biosynthetic pathways in Aspergillus nidulans (Trichocomaceae) that lead to a wide variety of chemical structures [2534]. Many of these compounds contain aromatic ring structures common to previously identified tau aggregation inhibitors. We therefore sought to determine whether A. nidulans secondary metabolites may also have tau aggregation inhibition activity.

We assessed the biological activity of 17 compounds using a standard arachidonic acid induction of tau aggregation in vitro [35] followed by a filter trap assay $[16,36]$ and electron microscopy $[37,38]$. The previously identified tau aggregation inhibitor emodin served as a positive control. Several of the compounds inhibited aggregation, and the inhibition by three of the compounds was reproducible and dose-dependent. We also assessed the effect of the compounds on tau's normal function of stabilizing microtubules using a fluorescence-based assay [39]. While the compounds reduced the activity of tau in a concentration-dependent manner, tau retained its ability to stimulate the polymerization of microtubules in the presence of the compounds, making them interesting candidate compounds for further development. Lastly, while two of the compounds are structurally similar to compounds that have been shown to inhibit tau aggregation, the third is quite different structurally and thus is the founding member of a new class of tau aggregation inhibitors. Interestingly, this compound is the precursor to the azaphilone chemical class of molecules, a class that includes compounds with lipoxygenase inhibitor activity [28], another activity of potential value in the treatment of dementia.

\section{Results}

$\nabla$

Because many A. nidulans secondary metabolites have chemical structures similar to previously identified inhibitors of tau aggregation, we sought to determine whether these compounds would have biological activity in inhibiting tau aggregation in vitro. We chose 17 compounds based on their preponderance of ring structures. These compounds include 8 anthraquinones, 5 xanthones, and 4 other types of metabolites ( Fig. 1). One compound (emodin) had been identified in an earlier study as an inhibitor of tau aggregation [16]. Tau polymerization was initiated in vitro using a standard arachidonic acid induction assay [35]. Each of the compounds, at a concentration of $200 \mu \mathrm{M}$, was preincubated with $2 \mu \mathrm{M}$ tau for $20 \mathrm{~min}$ before the addition of $75 \mu \mathrm{M}$ arachidonic ac- 


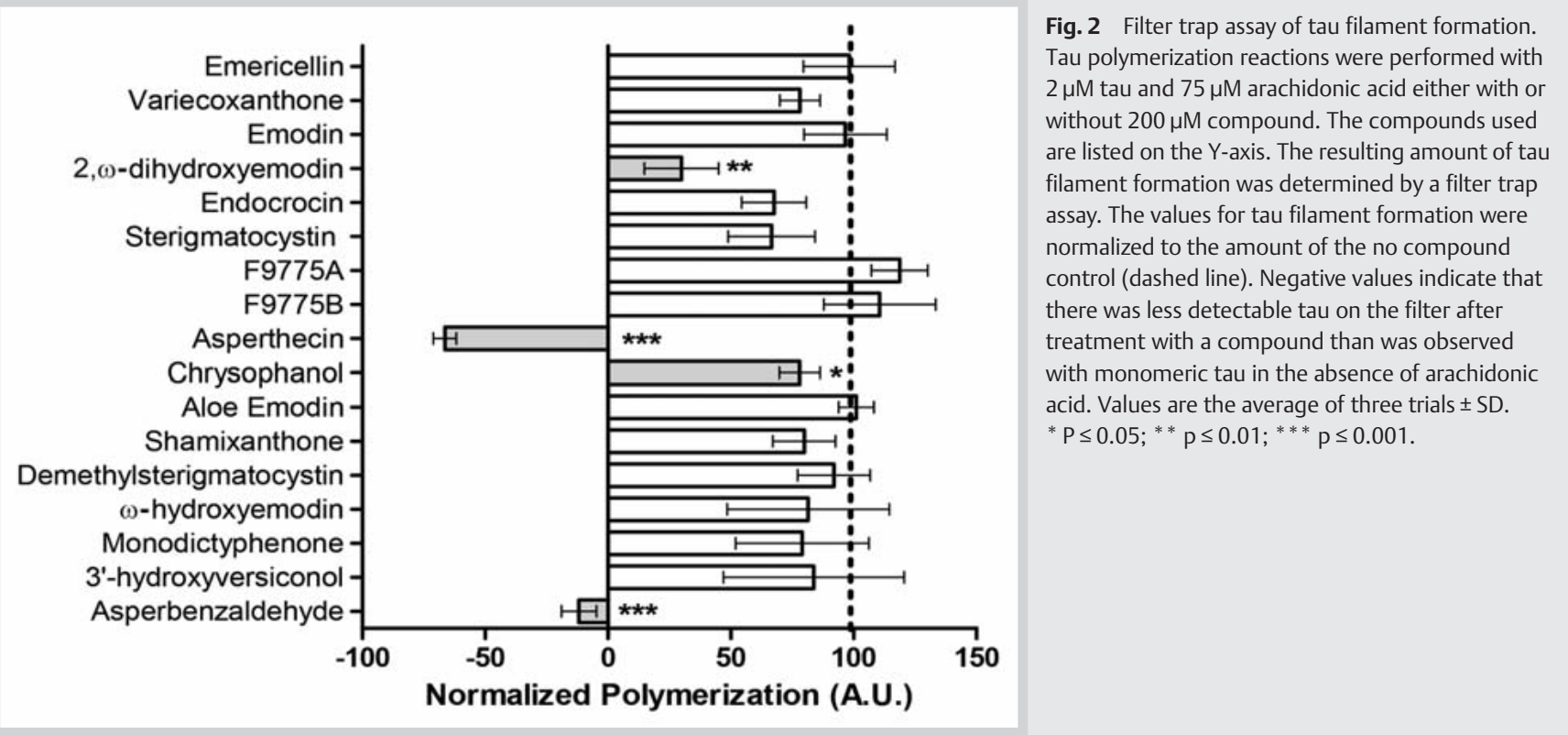

id. The amount of tau polymerization was determined using a filter trap assay $[16,36]$.

Variecoxanthone, 2, $\omega$-dihydroxyemodin, endocrocin, sterigmatocystin, asperthecin, chrysophanol, shamixanthone, and asperbenzaldehyde reduced the amount of tau aggregation ( $\mathbf{F i g . 2}$ ). Asperbenzaldehyde, asperthecin, and 2, $\omega$-dihydroxyemodin had the highest levels of inhibition. Although emodin inhibits tau aggregation when the glycosaminoglycan heparin is used as an inducer [16], it did not show appreciable inhibition in our assay. We have previously shown that arachidonic acid is a more potent inducer of tau aggregation than heparin [35], and we believe it is likely that arachidonic acid, coupled with the particular tau isoform we used, overwhelms the ability of emodin to inhibit tau aggregation (see Discussion).

We then sought to confirm the inhibition of tau aggregation using electron microscopy, another technique used to quantify tau aggregation reactions [40]. Asperthecin, asperbenzaldehyde, sterigmatocystin, and 2, $\omega$-dihydroxyemodin seemed to cause the largest reduction in tau aggregation in comparison with control reactions without a compound ( Fig. 3 ). We performed quantitative analysis of the filament lengths in the presence and absence of compounds. Filament lengths were placed into $50 \mathrm{~nm}$ bins. The first bin is $30-50 \mathrm{~nm}$ because it is difficult to distinguish tau particles below $30 \mathrm{~nm}$ in length from the background of the electron microscope grid. All filaments above $200 \mathrm{~nm}$ in length were binned together because this cutoff should represent particles retained by the filter trap assay. Inhibition reactions containing the positive control emodin, emericellin, variecoxanthone, 2, $\omega$-dihydroxyemodin, endocrocin, sterigmatocystin, F9775A, F9775B, asperthecin, aloe emodin, demethylsterigmatocystin, $\omega$-hydroxyemodin, monodictyphenone, 3'-hydroxyversiconol, and asperbenzaldehyde had filament length distributions that were distinct from reactions without a compound (० Fig. 4). These differences tended to include more objects in the 30$50 \mathrm{~nm}$ and $50-100 \mathrm{~nm}$ bins and fewer filaments in the $>200 \mathrm{~nm}$ bin. Asperbenzaldehyde had the greatest effect on filament formation with no filaments detected above $100 \mathrm{~nm}$ in length.

Many of the compounds reduced tau aggregation as measured by either the filter trap assay or quantitative electron microscopy, supporting our rationale for choosing this set of compounds. Also, four compounds showed a reduction in tau aggregation with both the filter trap assay and electron microscopy: 2, $\omega$-dihydroxyemodin, sterigmatocystin, asperthecin, and asperbenzaldehyde. We therefore sought to determine the $\mathrm{IC}_{50}$ for each compound. The $\mathrm{IC}_{50}$ for sterigmatocystin could not be determined because it did not demonstrate a consistent and reproducible concentration-dependent decrease in tau filament formation (data not shown). 2, $\omega$-Dihydroxyemodin, asperthecin, and asperbenzaldehyde gave reasonably consistent and reproducible concentration-dependent decreases in tau filament formation and had IC $_{50}$ values of $205 \pm 28 \mu \mathrm{M}, 39 \pm 2 \mu \mathrm{M}$, and $177 \pm 103 \mu \mathrm{M}$, respectively (๑ Fig. 5).

We also determined whether these three compounds would interfere with tau's normal function of stabilizing microtubules. Microtubule polymerization was monitored using DAPI fluorescence in the presence of tau with and without a compound ( Fig. 6). Although 2, $\omega$-dihydroxyemodin, asperthecin, and asperbenzaldehyde showed a dose-dependent reduction in tau's stabilization of microtubules, significant tubulin stabilization remained even at higher concentrations of inhibitors.

\section{Discussion}

$\nabla$

Tau filament formation is associated with many neurodegenerative diseases and is likely a significant contributor to the accompanying neuronal dysfunction and death. There are currently no available treatments to inhibit tau aggregation in Alzheimer's disease or other related dementias. There is great interest in identifying small molecules that may inhibit or reverse tau aggregation. Previous studies have identified several classes of compounds that show promise as aggregation inhibitors including anthraquinones, phenothiazines, and a benzothiazolidine derivative $[13,14,16]$. Many of the previously identified aggregation inhibitors share the common feature of containing aromatic ring structures. It is believed that these ring structures could interact with the $\beta$-structure that characterizes aggregation prone tau conformations thereby preventing the propagation of fila- 

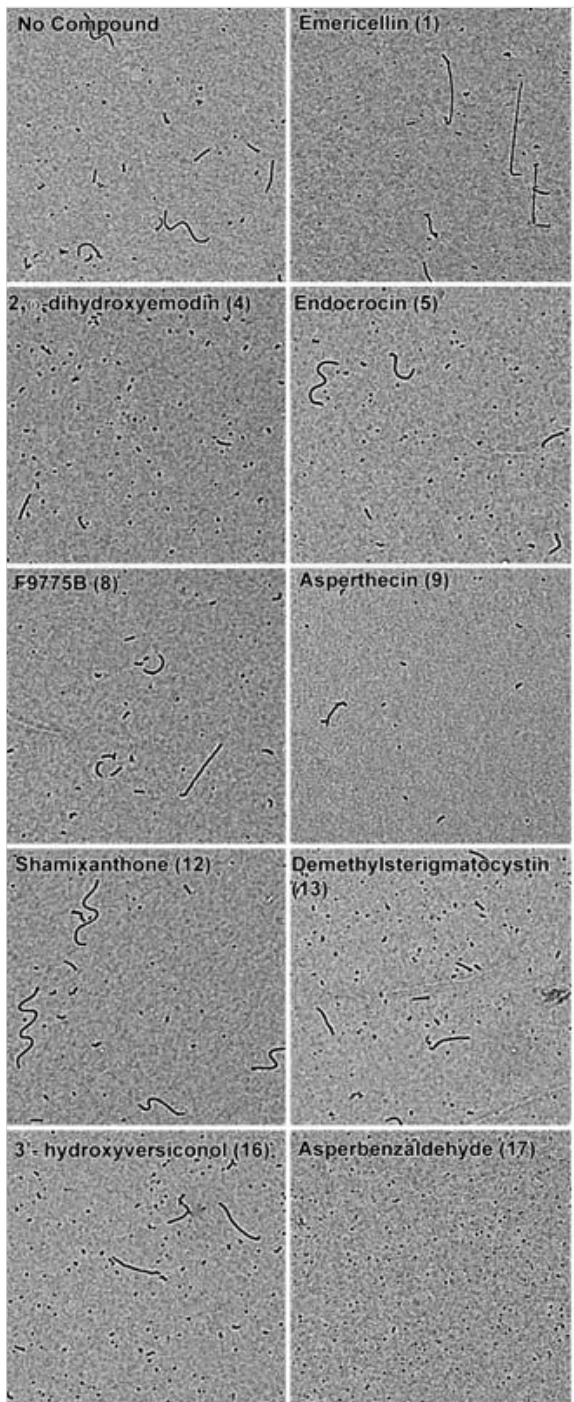
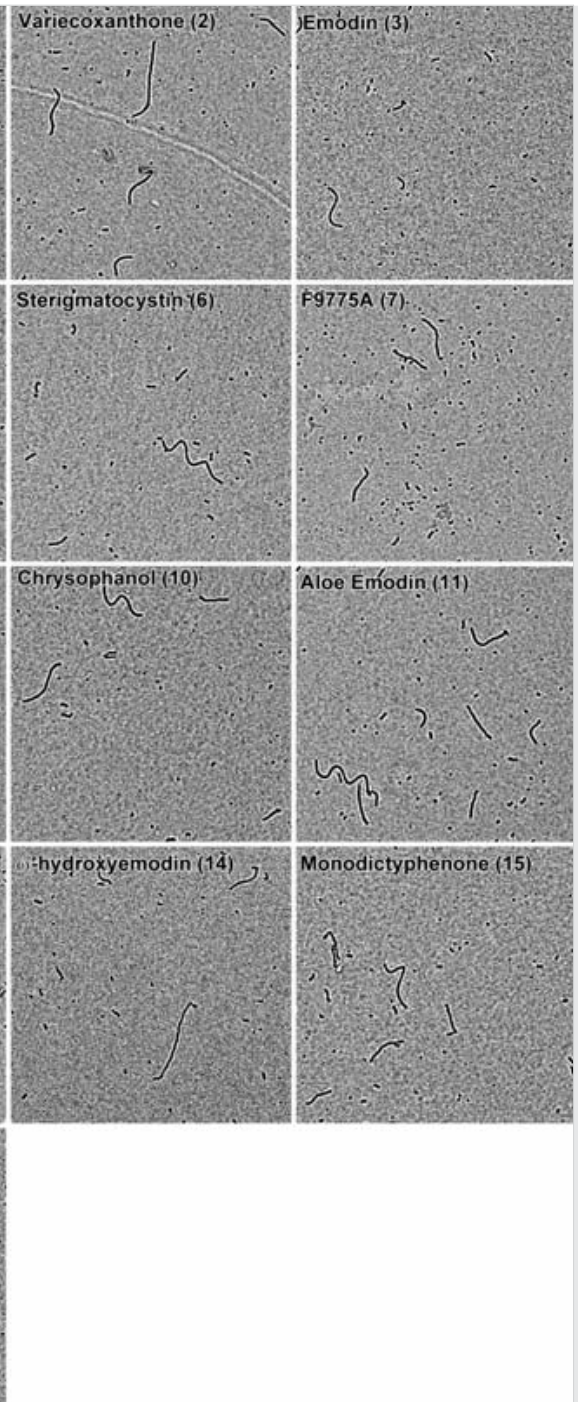

ment formation. While these compounds show promise, there remains a need for additional lead compounds for further development into potential therapeutics.

Many fungal natural products, including low molecular weight secondary metabolites, have been shown to have pharmacological properties such as acting as antibiotics, cholesterol synthesis inhibitors, immunosuppressants, and others [41]. Recent advances in genetic manipulation of silent secondary metabolite synthesis gene clusters have enhanced our capabilities to produce, purify and characterize fungal natural products and also allow the testing of these small molecules for biological or pharmacological activities. We have manipulated the genome of Aspergillus nidulans to produce a wide variety of natural products in amounts that allow ready purification [22,42]. Many of these small compounds have aromatic ring structures similar to those found in tau aggregation inhibitors.

$2, \omega$-Dihydroxyemodin and asperthecin belong to the anthraquinone class of compounds. Other anthraquinones have previously been shown to inhibit tau aggregation [16]. Notably, however, in this study both of these compounds proved to be more potent inhibitors of tau polymerization than emodin, which has been previously shown to be a strong inhibitor with an $\mathrm{IC}_{50}$ in the range of 1-5 $\mu \mathrm{M}$ [16]. Although our compounds were much more effective at inhibiting tau aggregation than emodin, it was surprising that
Fig. 3 EM images of polymerization reactions. Tau polymerization reactions were performed with $2 \mu \mathrm{M}$ tau and $75 \mu \mathrm{M}$ arachidonic acid either with or without $200 \mu \mathrm{M}$ compound. Aliquots of the reactions were prepared for negative stain electron microscopy. The individual images are labeled with the compound used.

their $\mathrm{IC}_{50}$ values, in the range of $40-200 \mu \mathrm{M}$, were much higher than those published for emodin. One likely explanation is that our assay conditions strongly drive aggregation and provide a particularly rigorous test for inhibition. The previous study was done using the glycosaminoglycan heparin sulfate as an inducer [16], and previous studies have shown that arachidonic acid induces approximately three times the amount of aggregation as heparin sulfate under similar conditions [35]. The previous emodin study also used 0N3R and 0N4R isoforms of tau [16], while our current study uses 2N4R tau. Our previous studies show that in the presence of arachidonic acid, 2N4R tau is much more prone to aggregation than the 0N3R and 0N4R isoforms [43]. These factors together could account for the differences in $\mathrm{IC}_{50}$ values and provide an explanation for why the $\mathrm{IC}_{50}$ values for our compounds are much higher than those previously reported even though they are more potent than emodin and, by inference, other previously reported tau aggregation inhibitors.

In our analysis, 2 of the 8 anthraquinones were active in blocking tau aggregation while none of the xanthone compounds were as effective. This could indicate the importance of the chemistry of the central ring. Anthraquinones contain 2 keto groups on their central ring, while xanthones contain a single keto group and an ether linkage. It is possible that the keto groups in the central ring are better aligned to interact with the beta-strand forming re- 


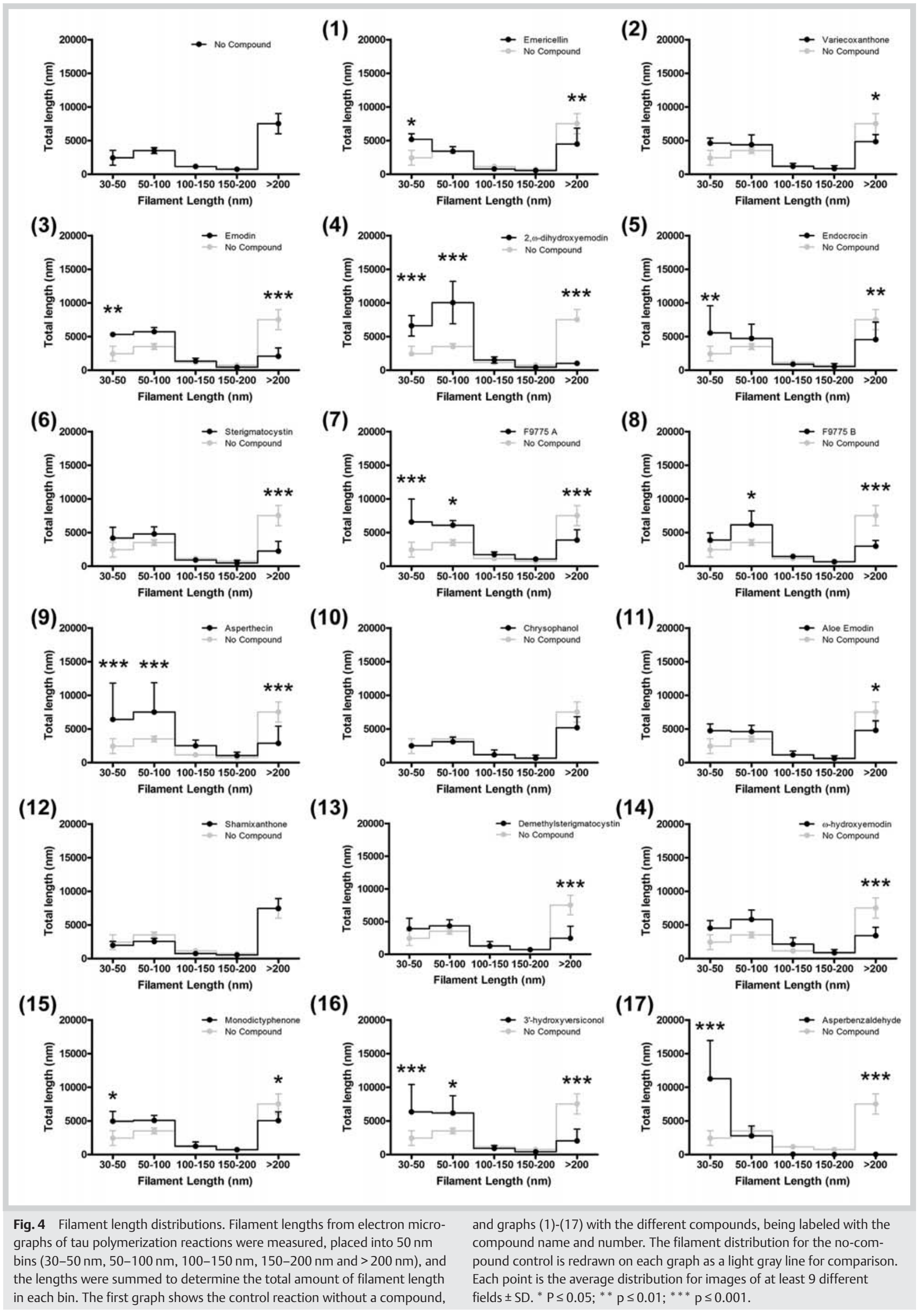



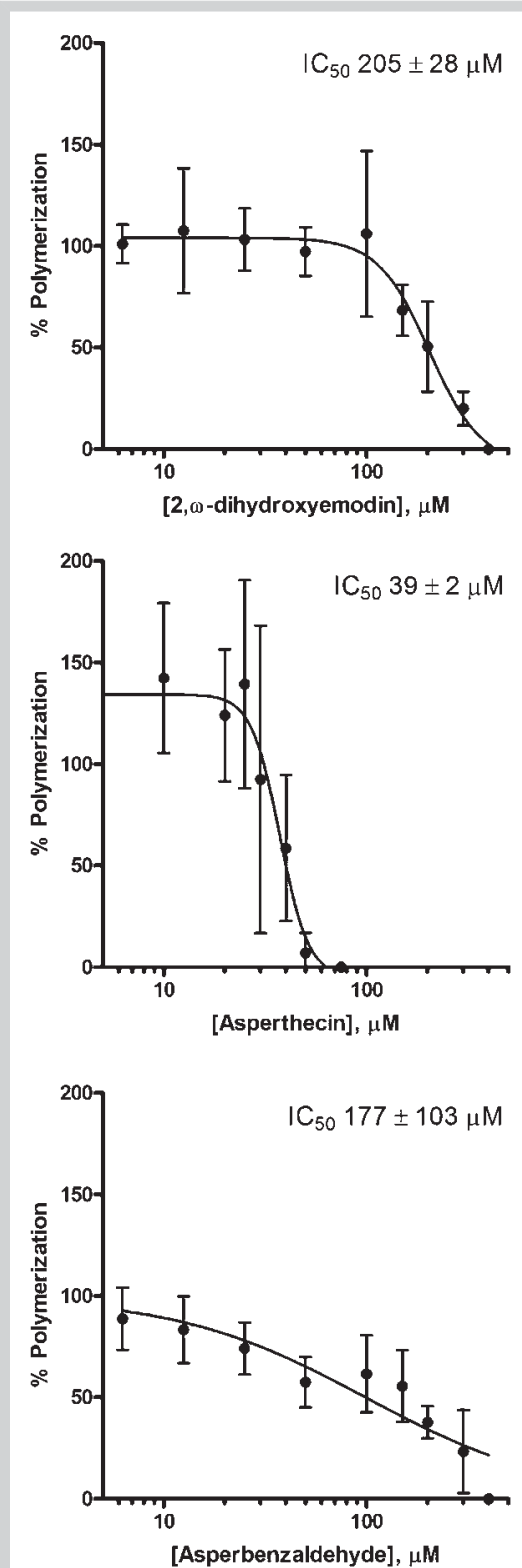

Fig. $5 \quad \mathrm{IC}_{50}$ determination. Polymerization reactions at $2 \mu \mathrm{M}$ tau and $75 \mu \mathrm{M}$ arachidonic acid were performed at several different concentrations of the compounds, and the resulting amount of filament formation was determined by a filter trap assay. The amount of polymerization was normalized to controls without a compound (100\%). The normalized data was plotted against the log of the inhibitor concentration and fit to a dose-response curve (solid line) to determine the $\mathrm{IC}_{50}$ for $\mathbf{A}$ 2, $\omega$-dihydroxyemodin, B asperthecin, and $\mathbf{C}$ asperbenzaldehyde. Data points are the average of three trials $\pm S D$. gions of tau than the xanthone ring. It is interesting that the compounds contain ring structures when considering that heparin sulfate also contains ring structures and arachidonic acid can adopt conformations in solution that could also be considered to be ring-like in shape. It is possible that the inhibitory compounds bind to tau in a manner similar to that of the inducers but block the addition of more molecules of tau.

Asperbenzaldehyde has a single aromatic ring and is a stable intermediate in the asperfuranone biosynthetic pathway. It is particularly interesting because, as an azaphilone intermediate, it is the founding member of a new class of compounds that inhibit tau aggregation. Azaphilones are a particularly interesting group of compounds because they exhibit a great variety of biologically important activities including antioxidant and anti-inflammatory activities (reviewed in [44]). Another interesting property of asperbenzaldehyde is that, while it has weak lipoxygenase- 1 (LOX-1) inhibitory activity, simple modifications convert it into a series of strong LOX-1 inhibitors [28]. Inhibition of LOX-1 may help to reduce fatty acid metabolites of arachidonic acid and do-
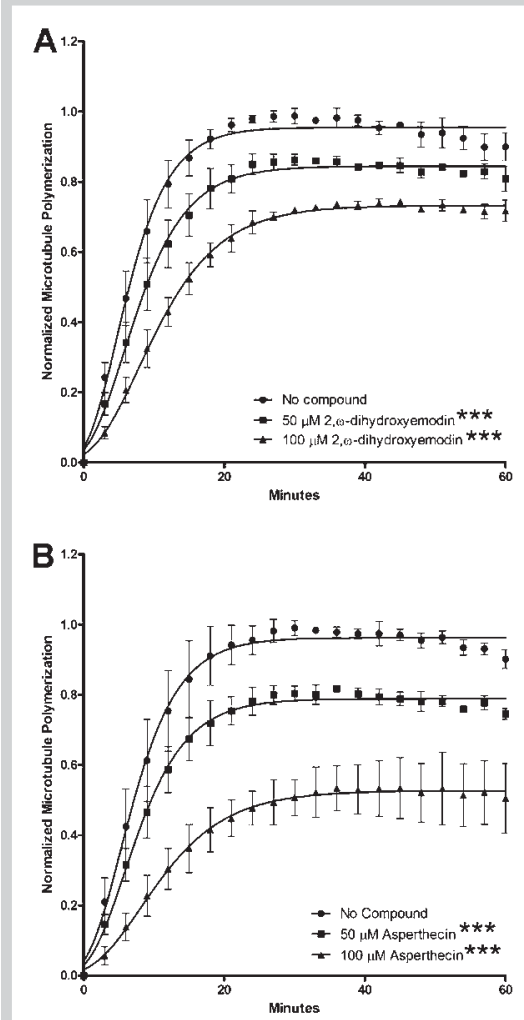

Fig. 6 Microtubule assembly. Tubulin was incubated with either tau protein alone $(\cdot)$ or tau in the presence of A 2, $\omega$-dihydroxyemodin, $\mathbf{B}$ asperthecin, or C asperbenzaldehyde at compound concentrations of $50 \mu \mathrm{M}(\mathbf{\square})$ or $100 \mu \mathrm{M}(\mathbf{\Lambda})$. Microtubule assembly was monitored by DAPI fluorescence ( $y$-axis) over time ( $x$-axis) and normalized to microtubule polymerization in the presence of paclitaxel. Every third time point is shown. Each point is the average of three independent trials \pm SD. The data are fit to a Gompertz growth curve (solid, dashed, and dotted lines for no compound, $50 \mu \mathrm{M}$ and $100 \mu \mathrm{M}$ compound, respectively). ${ }^{*} \mathrm{P} \leq 0.05$; ${ }^{* *} \mathrm{p} \leq 0.01 ;{ }^{* * *} \mathrm{p} \leq$ 0.001 .

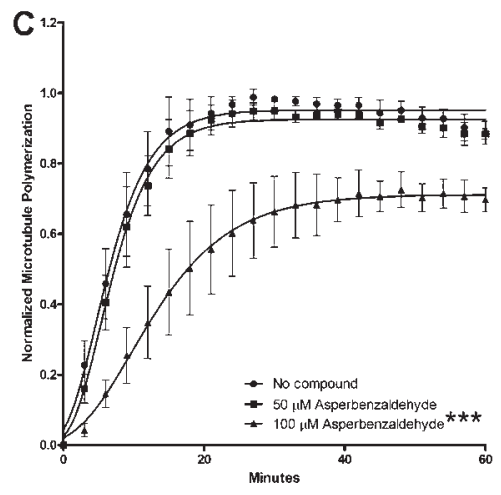

cosahexanoic acid that are elevated in Alzheimer's disease [45], and it is possible that derivatives of asperbenzaldehyde may have more than one positive therapeutic activity in tau dementias. One goal of tau therapeutics is that they should prevent undesirable tau aggregation while still retaining normal tau function of stabilizing the assembly of tubulin into microtubules. These compounds do reduce tau function but do not completely inhibit it. It is perhaps not surprising that some diminution occurs in the presence of compounds because the sequences likely responsible for tau aggregation are ${ }^{275}$ VQIINK ${ }^{280}$ and ${ }^{306} V_{Q} I_{V Y K}{ }^{311}$ which reside in the microtubule binding repeats $[10,11]$. If the compounds are interacting with these sequences as a mechanism for inhibiting tau aggregation, they would also likely have some impact on microtubule stabilization. It is encouraging that these compounds do allow some retention of tau function and thus are promising as future seed compounds to obtain derivatives with even greater ability to inhibit tau aggregation with fewer effects on tau's normal function of stabilizing microtubules. 
In conclusion, this report shows that selected secondary metabolites from fungi include seed compounds that may be modified for further therapeutic benefit. We have also provided another example of the wide application of fungal natural products. Lastly, we have identified an azaphilone precursor, asperbenzaldehyde, that inhibits both tau aggregation and LOX-1. The fact that asperbenzaldehyde has a quite different chemical structure from previously identified small molecule inhibitors of tau aggregation opens a new direction for tau inhibitor discovery.

\section{Materials and Methods \\ $\nabla$}

\section{Chemicals and reagents}

Full length 2N4R tau (441 amino acids) was expressed in E. coli and purified as described previously [46]. Arachidonic acid (ARA) was purchased from Cayman Chemicals. Compounds were purified from Aspergillus nidulans as described previously [28, 29, $31,32,47,48$ ] from a single peak of HPLC chromatography, and purity was estimated by NMR (see Table $1 S$ and Fig. 1S, Supporting Information).

\section{ARA-induced tau polymerization reactions}

$2 \mu \mathrm{M}$ recombinant tau protein was incubated in polymerization buffer which contained $10 \mathrm{mM}$ HEPES (pH7.64), $5 \mathrm{mM}$ DTT, $100 \mathrm{mM} \mathrm{NaCl}, 0.1 \mathrm{mM}$ EDTA, and 3.75\% ethanol. Compounds dissolved in DMSO were added to the tau solution at final concentrations of $200 \mu \mathrm{M}, 100 \mu \mathrm{M}, 50 \mu \mathrm{M}$, or $25 \mu \mathrm{M}$ as described in Results. Compounds were allowed to incubate with tau for $20 \mathrm{~min}$ before the addition of $75 \mu \mathrm{M}$ arachidonic acid to initiate tau filament formation [35]. The reactions were allowed to proceed at room temperature for $16 \mathrm{~h}$ before analysis.

\section{Filter trap assay}

Tau polymerization reactions, as described above, were diluted to $20 \mathrm{ng} / 300 \mu \mathrm{L}$ in TBS and passed through a pre-wetted nitrocellulose membrane (Bio-Rad Laboratories) using vacuum force in a dot-blot apparatus (Bio-Rad Laboratories). The membranes were washed thrice with TBS-0.05\% Tween 20 (TBST) and then blocked in $5 \%$ nonfat dry milk in TBST for $1 \mathrm{~h}$. The membranes were then incubated with primary antibody mixture [Tau 5 [49] at $1: 50000$ dilution, Tau 12 [50] at $1: 250000$ dilution, and Tau 7 [51] at $1: 250000$ dilution; antibodies were a kind gift from Dr. Lester I. Binder] overnight at $4^{\circ} \mathrm{C}$. Membranes were washed thrice in TBST and incubated with secondary antibody, HRP-linked goat antimouse IgG (Thermo Scientific), for $1 \mathrm{~h}$ at room temperature. The membranes were washed twice in TBS-Tween buffer, and a final wash was made with TBS. The blot was developed using an ECL (enhanced chemiluminescence) Western blotting analysis system (GE Healthcare). Images were captured with a Kodak Image Station 4000R and were quantified using the histogram function of Adobe Photoshop 7.0. Statistical analyses were performed using 1-way ANOVA with Dunnett's multiple comparison test to compare the triplicate values to control values.

\section{Transmission electron microscopy}

Polymerization reaction samples were diluted $1: 10$ in polymerization buffer and fixed with $2 \%$ glutaraldehyde for 5 min. $10 \mu \mathrm{L}$ of each sample was added to a Formvar carboncoated grid for $1 \mathrm{~min}$. The grid was blotted on filter paper, washed with water, blotted, washed with $2 \%$ uranyl acetate and blotted before staining with $2 \%$ uranyl acetate for 1 min followed by a final blotting on filter paper. The grids were examined with a Technai F20 XT field emission transmission electron microscope (FEI Co.). Images were taken with the Gatan Digital Micrograph imaging system. The images were collected at a magnification of $3600 \times$. The filaments were quantified using Image-Pro Plus 6.0 software [35]. The perimeter of the filaments was determined, and the length was obtained by dividing the perimeter in half. For quantitative analysis, filament lengths were placed into bins as described in Results. Statistical analyses were performed using two-way ANOVA with Bonferroni post-tests to compare replicate means for each bin size with the no compound data serving as reference values.

\section{Tubulin polymerization assay}

Polymerization of tubulin was measured using a tubulin polymerization assay kit (Cytoskeleton, Inc.). The reactions were measured in 96-well Costar black polystyrene flat-bottom plates (Corning, Inc.). Each well contained porcine tubulin at $2 \mathrm{mg} / \mathrm{mL}$ and GTP at $1 \mathrm{mM}$ in $80 \mathrm{mM}$ PIPES buffer (pH 6.9) with $2 \mathrm{mM}$ $\mathrm{MgCl}_{2}$ and $0.5 \mathrm{mM}$ EGTA. Tau was added to the wells at a concentration of $1 \mu \mathrm{M}$, and the test compounds diluted in DMSO were added at a final concentration of $200 \mu \mathrm{M}$. An equal amount of DMSO was added to each of the wells. Another well containing $1 \mu \mathrm{M}$ paclitaxel (99.1\% pure; Cytoskeleton, Inc.) and tubulin served as a positive control for polymerization and as a standard for normalizing results for each of the experiments (not shown). Control reactions containing only buffer without either tau or a compound, and buffer with the individual test compounds but without tau were also performed (not shown). Reaction plates were placed at $37^{\circ} \mathrm{C}$ and shaken for $5 \mathrm{~s}$ in a FlexStation II fluorometer (Molecular Devices Corp.). The fluorescence was measured at a constant interval of 1 minute for an hour with an excitation wavelength $\left(\lambda_{\mathrm{ex}}\right)$ of $355 \mathrm{~nm}$ and an emission wavelength $\left(\lambda_{\mathrm{em}}\right)$ of $455 \mathrm{~nm}$. This gave 60 readings for every well sample. The resulting data were normalized to the amount of microtubule polymerization observed in the presence of tau without a compound and fit to the Gompertz equation [43]. Experiments were performed in triplicate and averaged. Statistical analyses and individual growth parameters can be found in Table 2S, Supporting Information.

\section{Supporting information}

Data on the estimated purity of the tested compounds based on ${ }^{1} \mathrm{H}$ NMR spectra and the spectrum of emericellin used to determine compound purity are available as Supporting Information.

\section{Acknowledgements}

$\nabla$

The Tau5, Tau12, and Tau7 antibodies were kindly provided by Lester I. Binder (Northwestern University). This work was supported in part by grant P01-GM084077 from the National Institute of General Medical Sciences (C.C.C.W. and B.R.O.) and by the H. L. Snyder Medical Foundation (B.R.O. and T.C.G.).

\section{Conflict of Interest \\ $\nabla$}

The authors declare no conflict of interest. 
Affiliations

${ }^{1}$ Department of Molecular Biosciences, University of Kansas, Lawrence, KS, USA

2 Department of Pharmacology and Pharmaceutical Sciences, School of Pharmacy, University of Southern California, Los Angeles, CA, USA

${ }^{3}$ Graduate Institute of Pharmaceutical Science, Chia Nan University School of Pharmacy and Science, Tainan, Taiwan

${ }^{4}$ Department of Chemistry, Dornsife College of Letters, Arts, and Sciences, University of Southern California, Los Angeles, CA, USA

\section{References}

1 Arriagada PV, Growdon JH, Hedley-Whyte ET, Hyman BT. Neurofibrillary tangles but not senile plaques parallel duration and severity of Alzheimer's disease. Neurology 1992; 42: 631-639

2 Goedert M, Crowther RA, Spillantini MG. Tau mutations cause frontotemporal dementias. Neuron 1998; 21: 955-958

3 Goedert M, Ghetti B, Spillantini MG. Tau gene mutations in frontotemporal dementia and parkinsonism linked to chromosome 17 (FTDP-17). Their relevance for understanding the neurogenerative process. Ann N Y Acad Sci 2000; 920: 74-83

4 Ko LW, DeTure M, Sahara N, Chihab R, Vega IE, Yen SH. Recent advances in experimental modeling of the assembly of tau filaments. Biochim Biophys Acta 2005; 1739: 125-139

5 Brunden KR, Ballatore C, Crowe A, Smith 3rd AB, Lee VM, Trojanowski JQ. Tau-directed drug discovery for Alzheimer's disease and related tauopathies: a focus on tau assembly inhibitors. Exp Neurol 2010; 223: 304-310

6 Martin L, Latypova X, Terro F. Post-translational modifications of tau protein: implications for Alzheimer's disease. Neurochem Int 2011; 58: $458-471$

7 Martin L, Latypova X, Wilson CM, Magnaudeix A, Perrin ML, Terro F. Tau protein phosphatases in Alzheimer's disease: the leading role of PP2A. Ageing Res Rev 2013; 12: 39-49

8 Martin L, Latypova X, Wilson CM, Magnaudeix A, Perrin ML, Yardin C, Terro F. Tau protein kinases: involvement in Alzheimer's disease. Ageing Res Rev 2013; 12: 289-309

9 Mondragon-Rodriguez S, Perry G, Luna-Munoz J, Acevedo-Aquino M, Williams S. Phosphorylation of tau protein at sites Ser is one of the earliest events in Alzheimer's disease and Down syndrome. Neuropathol Appl Neurobiol 2013, epub ahead of print; DOI: 10.1111/nan.12084; in press

10 von Bergen M, Barghorn S, Biernat J, Mandelkow EM, Mandelkow E. Tau aggregation is driven by a transition from random coil to beta sheet structure. Biochim Biophys Acta 2005; 1739: 158-166

11 von Bergen M, Friedhoff P, Biernat J, Heberle J, Mandelkow E. Assembly of tau protein into Alzheimer paired helical filaments depends on a local sequence motif (306VQIVYK311) forming beta structure. Proc Natl Acad Sci U S A 2000; 97: 5129-5134

12 Taniguchi S, Suzuki N, Masuda M, Hisanaga S, Iwatsubo T, Goedert M, Hasegawa $M$. Inhibition of heparin-induced tau filament formation by phenothiazines, polyphenols, and porphyrins. J Biol Chem 2005; 280: 7614-7623

13 Wischik CM, Edwards PC, Lai RY, Roth M, Harrington CR. Selective inhibition of Alzheimer disease-like tau aggregation by phenothiazines. Proc Natl Acad Sci USA 1996; 93: 11213-11218

14 Chirita C, Necula M, Kuret J. Ligand-dependent inhibition and reversal of tau filament formation. Biochemistry 2004; 43: 2879-2887

15 Necula M, Chirita CN, Kuret J. Cyanine dye N744 inhibits tau fibrillization by blocking filament extension: implications for the treatment of tauopathic neurodegenerative diseases. Biochemistry 2005; 44: 10227-10237

16 Pickhardt M, Gazova Z, von Bergen M, Khlistunova I, Wang Y, Hascher A, Mandelkow EM, Biernat J, Mandelkow E. Anthraquinones inhibit tau aggregation and dissolve Alzheimer's paired helical filaments in vitro and in cells. J Biol Chem 2005; 280: 3628-3635

17 Khlistunova I, Biernat J, Wang Y, Pickhardt M, von Bergen M, Gazova Z, Mandelkow E, Mandelkow EM. Inducible expression of Tau repeat domain in cell models of tauopathy: aggregation is toxic to cells but can be reversed by inhibitor drugs. J Biol Chem 2006; 281: 1205-1214

18 Pickhardt M, Larbig G, Khlistunova I, Coksezen A, Meyer B, Mandelkow $E M$, Schmidt B, Mandelkow E. Phenylthiazolyl-hydrazide and its derivatives are potent inhibitors of tau aggregation and toxicity in vitro and in cells. Biochemistry 2007; 46: 10016-10023

19 Bulic B, Pickhardt M, Khlistunova I, Biernat J, Mandelkow EM, Mandelkow E, Waldmann H. Rhodanine-based tau aggregation inhibitors in cell models of tauopathy. Angew Chem Int Ed Engl 2007; 46: 92159219

20 Crowe A, Ballatore C, Hyde E, Trojanowski JQ Lee VM. High throughput screening for small molecule inhibitors of heparin-induced tau fibril formation. Biochem Biophys Res Commun 2007; 358: 1-6

21 Crowe A, Huang W, Ballatore C, Johnson RL, Hogan AM, Huang R, Wichterman J, McCoy J, Huryn D, Auld DS, Smith 3rd AB, Inglese J, Trojanowski $J Q$ Austin CP, Brunden KR, Lee VM. Identification of aminothienopyridazine inhibitors of tau assembly by quantitative high-throughput screening. Biochemistry 2009; 48: 7732-7745

22 Oakley CE, Edgerton-Morgan H, Oakley BR. Tools for manipulation of secondary metabolism pathways: rapid promoter replacements and gene deletions in Aspergillus nidulans. Methods Mol Biol 2012; 944: 143-161

23 Szewczyk E, Nayak T, Oakley CE, Edgerton H, Xiong Y, Taheri-Talesh N, Osmani SA, Oakley BR. Fusion PCR and gene targeting in Aspergillus nidulans. Nat Protoc 2006; 1: 3111-3120

24 Nayak T, Szewczyk E, Oakley CE, Osmani A, Ukil L, Murray SL, Hynes MJ, Osmani SA, Oakley BR. A versatile and efficient gene-targeting system for Aspergillus nidulans. Genetics 2006; 172: 1557-1566

25 Soukup AA, Chiang YM, Bok JW, Reyes-Dominguez Y, Oakley BR, Wang CC, Strauss J, Keller NP. Overexpression of the Aspergillus nidulans histone 4 acetyltransferase EsaA increases activation of secondary metabolite production. Mol Microbiol 2012; 86: 314-330

26 Yeh HH, Chiang YM, Entwistle R, Ahuja M, Lee KH, Bruno KS, Wu TK, Oakley $B R$, Wang CC. Molecular genetic analysis reveals that a nonribosomal peptide synthetase-like (NRPS-like) gene in Aspergillus nidulans is responsible for microperfuranone biosynthesis. Appl Microbiol Biotechnol 2012; 96: 739-748

27 Lo HC, Entwistle R, Guo CJ, Ahuja M, Szewczyk E, Hung JH, Chiang YM, Oakley BR, Wang CC. Two separate gene clusters encode the biosynthetic pathway for the meroterpenoids austinol and dehydroaustinol in Aspergillus nidulans. J Am Chem Soc 2012; 134: 4709-4720

28 Somoza AD, Lee KH, Chiang YM, Oakley BR, Wang CC. Reengineering an azaphilone biosynthesis pathway in Aspergillus nidulans to create lipoxygenase inhibitors. Org Lett 2012; 14: 972-975

29 Sanchez JF, Entwistle R, Hung JH, Yaegashi J, Jain S, Chiang YM, Wang CC, Oakley BR. Genome-based deletion analysis reveals the prenyl xanthone biosynthesis pathway in Aspergillus nidulans. J Am Chem Soc 2011: 133: 4010-4017

30 Sanchez JF, Chiang YM, Szewczyk E, Davidson AD, Ahuja M, Elizabeth Oakley C, Woo Bok J, Keller N, Oakley BR, Wang CC. Molecular genetic analysis of the orsellinic acid/F9775 gene cluster of Aspergillus nidulans. Mol Biosyst 2010; 6: 587-593

31 Chiang YM, Szewczyk E, Davidson AD, Entwistle R, Keller NP, Wang CC, Oakley BR. Characterization of the Aspergillus nidulans monodictyphenone gene cluster. Appl Environ Microbiol 2010; 76: 2067-2074

32 Chiang YM, Szewczyk E, Davidson AD, Keller N, Oakley BR, Wang CC. A gene cluster containing two fungal polyketide synthases encodes the biosynthetic pathway for a polyketide, asperfuranone, in Aspergillus nidulans. J Am Chem Soc 2009; 131: 2965-2970

33 Szewczyk E, Chiang YM, Oakley CE, Davidson AD, Wang CC, Oakley BR. Identification and characterization of the asperthecin gene cluster of Aspergillus nidulans. Appl Environ Microbiol 2008; 74: 7607-7612

34 Chiang YM, Szewczyk E, Nayak T, Davidson AD, SanchezJF, Lo HC, Ho WY, Simityan H, Kuo E, Praseuth A, Watanabe K, Oakley BR, Wang CC. Molecular genetic mining of the Aspergillus secondary metabolome: discovery of the emericellamide biosynthetic pathway. Chem Biol 2008; 15: 527-532

35 Carlson SW, Branden M, Voss K, Sun Q Rankin CA, Gamblin TC. A complex mechanism for inducer mediated tau polymerization. Biochemistry 2007; 46: 8838-8849

36 Chang E, Kuret J. Detection and quantification of tau aggregation using a membrane filter assay. Anal Biochem 2008; 373: 330-336

37 Combs B, Gamblin TC. FTDP-17 tau mutations induce distinct effects on aggregation and microtubule interactions. Biochemistry 2012; 51: 8597-8607

38 King ME, Gamblin TC, Kuret J, Binder LI. Differential assembly of human tau isoforms in the presence of arachidonic acid. J Neurochem 2000; 74: $1749-1757$

39 Barron DM, Chatterjee SK, Ravindra R, Roof R, Baloglu E, Kingston DG, Bane $S$. A fluorescence-based high-throughput assay for antimicrotubule drugs. Anal Biochem 2003; 315: 49-56

40 Necula M, Kuret J. Electron microscopy as a quantitative method for investigating tau fibrillization. Anal Biochem 2004; 329: 238-246 
41 Keller NP, Turner G, Bennett JW. Fungal secondary metabolism - from biochemistry to genomics. Nat Rev Microbiol 2005; 3: 937-947

42 Sanchez JF, Somoza AD, Keller NP, Wang CC. Advances in Aspergillus secondary metabolite research in the post-genomic era. Nat Prod Rep 2012; 29: 351-371

43 Combs B, Voss K, Gamblin TC. Pseudohyperphosphorylation has differential effects on polymerization and function of tau isoforms. Biochemistry 2011; 50: 9446-9456

44 Osmanova N, Schultze W, Ayoub N. Azaphilones: a class of fungal metabolites with diverse biological activities. Phytochem Rev 2010; 9: 315-342

45 Chu J, Pratico D. Pharmacologic blockade of 5-lipoxygenase improves the amyloidotic phenotype of an Alzheimer's disease transgenic mouse model involvement of gamma-secretase. Am J Pathol 2011; 178: $1762-1769$

46 Rankin CA, Sun $Q$ Gamblin TC. Pseudo-phosphorylation of tau at Ser202 and Thr205 affects tau filament formation. Brain Res Mol Brain Res 2005; 138: 84-93
47 Ahuja M, Chiang YM, Chang SL, Praseuth MB, Entwistle R, Sanchez JF, Lo $H C$, Yeh HH, Oakley BR, Wang CC. Illuminating the diversity of aromatic polyketide synthases in Aspergillus nidulans. J Am Chem Soc 2012; 134: 8212-8221

48 Bok JW, Chiang YM, Szewczyk E, Reyes-Dominguez Y, Davidson AD, Sanchez JF, Lo HC, Watanabe K, Strauss J, Oakley BR, Wang CC, Keller NP. Chromatin-level regulation of biosynthetic gene clusters. Nat Chem Biol 2009; 5: 462-464

49 LoPresti P, Szuchet S, Papasozomenos SC, Zinkowski RP, Binder LI. Functional implications for the microtubule-associated protein tau: localization in oligodendrocytes. Proc Natl Acad Sci USA 1995; 92: 1036910373

50 Horowitz PM, Patterson KR, Guillozet-Bongaarts AL, Reynolds MR, Carroll CA, Weintraub ST, Bennett DA, Cryns VL, Berry RW, Binder LI. Early $\mathrm{N}$-terminal changes and caspase-6 cleavage of tau in Alzheimer's disease. J Neurosci 2004; 24: 7895-7902

51 Horowitz PM, Lapointe N, Guillozet-Bongaarts AL, Berry RW, Binder LI. $\mathrm{N}$-terminal fragments of tau inhibit full-length tau polymerization in vitro. Biochemistry 2006; 45: 12 859-12866 